\title{
HIGH POWER HIGH FREQUENCY SOFT SWITCHING CONVERTER USING SERIAL CONNECTED SWITCHES
}

\author{
N. Lapassat ${ }^{\star}$, D Chatroux ${ }^{\star}$, D. Lafore ${ }^{\star *}$, J.F. Villard ${ }^{\star}$ \\ ${ }^{*}$ Commissariat à l'Energie Atomique, France. \\ ${ }^{\star \star}$ CEGEMA - ESIM, France.
}

Keywords: high power high frequency switch mode converter, serial connection, soft switching.

\begin{abstract}
This paper presents a chopper topology suitable for high voltage, with a switch made of serial connected power devices. Serial connection of power components encounters two major problems in hard switching : voltage unbalance and overcurrents at turn on due to parasitic capacitances to ground. The proposed topology takes advantage of soft switching to reduce both problems by a suitable utilisation of inductors and capacitors. A converter tested at $9 \mathrm{kV}$ input voltage is also presented.
\end{abstract}




\title{
HIGH POWER HIGH FREQUENCY SOFT SWITCHING CONVERTER USING SERIAL CONNECTED SWITCHES
}

\author{
N. Lapassat*, D Chatroux*, D. Lafore**, J.F. Villard* \\ *Commissariat à l'Energie Atomique, France. \\ ${ }^{\star \star}$ CEGEMA - ESIM, France.
}

Keywords: high power high frequency switch mode converter, serial connection, soft switching.

\section{INTRODUCTION}

In the field of high voltage power conversion, low or medium voltage inverters are generally used with high voltage transformers [1]. This article presents a high voltage chopper supplied directly by a high voltage source. At the same power level, higher input voltage means lower current stress in the switches, making realisation on printed board easier.

Such a solution requires serial connection to realise the high voltage switch. To reach a switching frequency of several tens of $\mathrm{kHz}$, MOS or IGBT are used. Serial connection of power components encounters two major problems in hard switching : voltage unbalance and overcurrents due to parasitic capacitances to ground during turn on. As the number of stages connected in series increases, those problems become more crucial.

Voltage unbalance is mainly due to differential delay time of drive circuit (switching on and off phases) and speed scattering (switching off phases). Synchronous orders and equal switching speeds are necessary to obtain a good voltage sharing [2]. Otherwise, active clamping can be used for grid components like IGBT [3]. During the off state, scattering of leakage current between semiconductors gives rise to significant voltage unbalance. Sharing resistor can be added in parallel of each switches for current balancing. At high frequency, they are no more needed [4]. Parasitic overcurrents in hard commutation, for high voltage low current switches using serial connection, is one of the main problems [2].

Soft switching can be used to reduce switching losses, voltage unbalance and parasitic overcurrents. This article present a converter combining serial connection with soft switching.

\section{SOFT SWITCHING TOPOLOGY}

The chosen soft switching topology is presented on fig. 1 [5]. Its main advantages are :

- Constant frequency.

- The two switches are triggered simultaneously.

- Soft switching conditions are simple to cope with. 


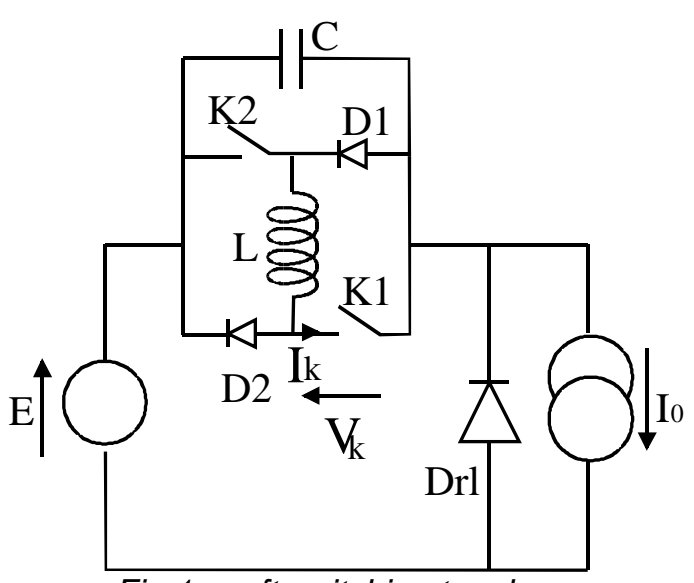

Fig.1 : soft switching topology.

\section{Principle of operation}

This converter works at constant frequency. Switches K1 and K2 are triggered simultaneously, simplifying driving problems.

At turn on, diodes $D_{1}$ and $D_{2}$ are on so that the inductor is in series with $\mathrm{K} 1$ and K2. At turn off, diodes $\mathrm{D}_{1}$ and $D_{2}$ are off and the capacitor is in parallel with $\mathrm{K} 1$ and $\mathrm{K} 2$. Thus, both commutation can be losseless.

Fig. 2 presents state plane analysis of the converter. There is no zero crossing for capacitor voltage and inductor current, and so there is no condition on the value of $x=\frac{I_{0}}{E} \sqrt{\frac{L}{C}}$.

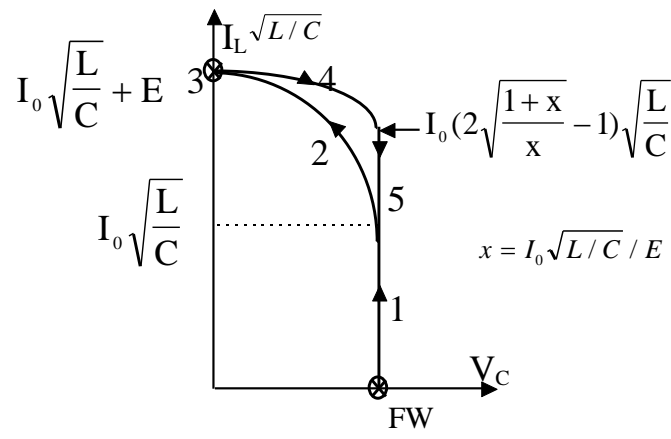

Fig. :2 : State plane analysis.

Fig. 3 exhibits capacitor's voltage and inductor's current waveforms, while fig. 4 presents voltage and current for K1 and K2.

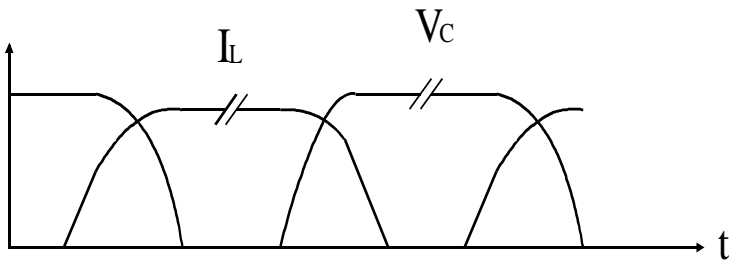

Fig 3 : Inductor's current and capacitor's voltage.

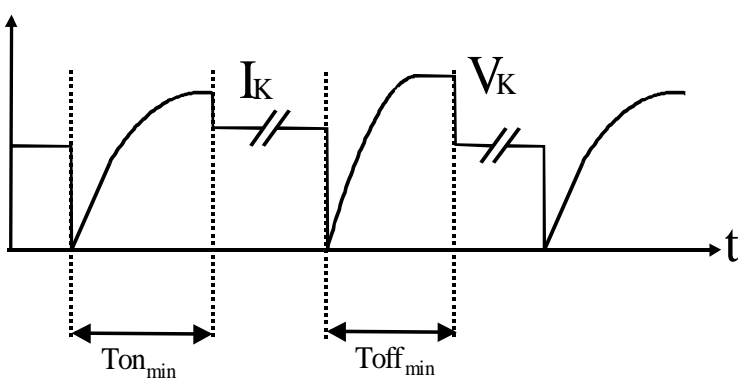

Fig. 4 : Voltage and current for $K 1$ and $K 2$.

\section{Soft switching limit}

One of the advantages of that topology is that soft switching conditions are easy to take into account: switches just have to respect minimum on and off time Ton min and Toff $\min$ (cf. fig. 4), so that limiting duty cycle in a suitable range allows soft switching. Moreover, if this is not respected, switches get into hard commutation cell conditions (cf. fig.5). This is interesting at light load: if Ton<Tonmin, the hard turn off just increases losses (which is not critical because conduction losses are then lower) with a moderate overvoltage caused by stray inductances.

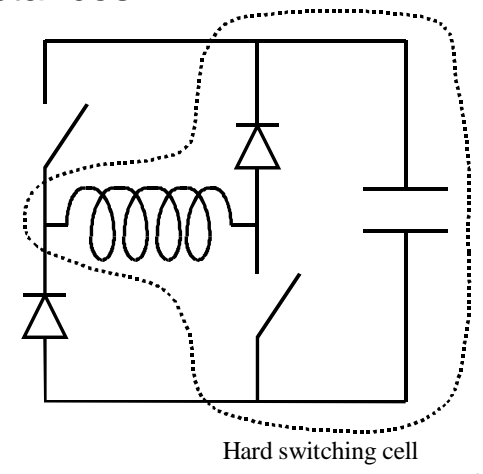

Fig 5 : Equivalent circuit in case of non respect of soft switching conditions. 


\section{USE EN SERIAL CONNECTION}

\section{High voltage switch}

Fig. 6 presents a suitable way of using the soft switching topology in serial connection.

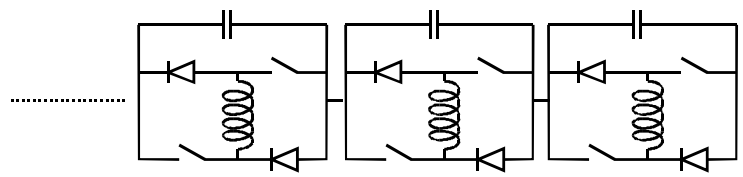

Fig 6 : high voltage switch.

This way of connecting switches allows distribution of inductors and capacitors among the devices. This is suitable to limit problems of voltage unbalance and parasitic overcurrents.

\section{Voltage unbalance}

At turn on, when a switch is late, it is submitted to an overvoltage. Here, resonant capacitors limit the overvoltage to low value: the voltage rise across a late stage is made by charging its capacitance with a current which is limited by inductances of others stages.

At turn off, capacitors limit and equalise $\mathrm{dV} / \mathrm{dt}$ of each stage so that overvoltages are moderate.

If a voltage unbalance can be kept at a low value on one cycle, it has an effect on following cycles : a stage with a higher capacitor voltage will get a higher inductor current, and then a higher voltage and so on. Fig. 7 show SPICE simulation of two stages in series. At each cycle, the first one turn on 100 ns after the second one. The capacitor voltage's unbalance increases during several cycles and then reaches a fixed value.
Fig. 8 shows a oscillogramm of voltages across capacitors for two serial connected stages, in a chopper configuration test. Voltage unbalance is $5 \%$, which is negligible (below tolerance on capacitance).

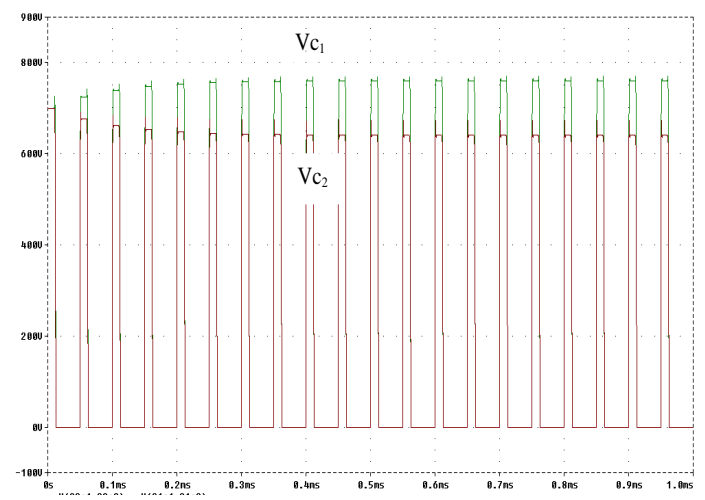

Fig. 7 : Evolution of a capacitor voltage unbalance due to a cyclical early turn on.

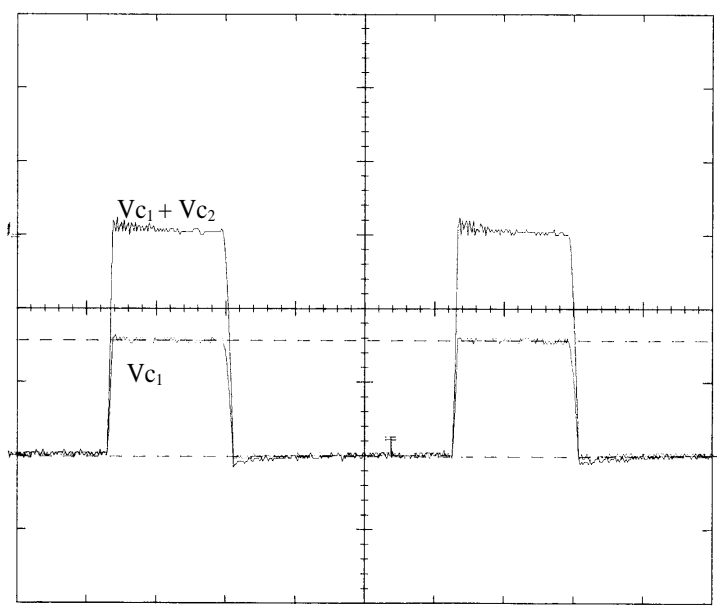

Fig. 8 : Voltage across two stages in series

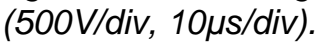

\section{Parasitic overcurrents}

At turn on, distributed inductors limit overcurrents due to parasitic capacitances to earth [6]. Inductors are distributed every two switches (cf. fig. 9). 


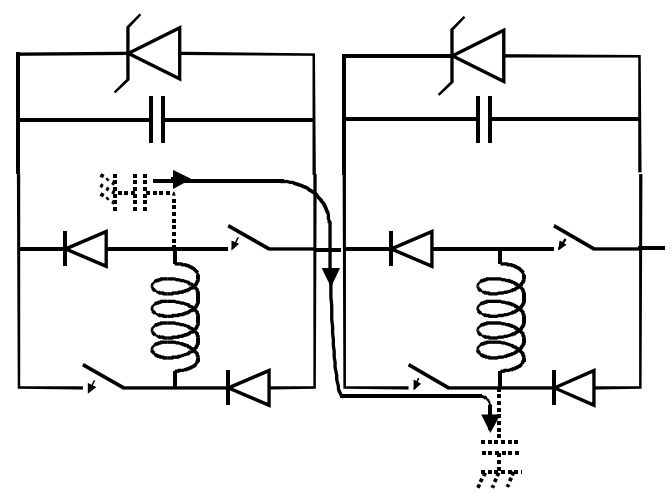

Fig. 9 : Parasitic current at turn on.

This converter allows the recycling of energy stored in parasitic capacitances to earth, as it is added to energy stored in resonant capacitors (cf. fig ).

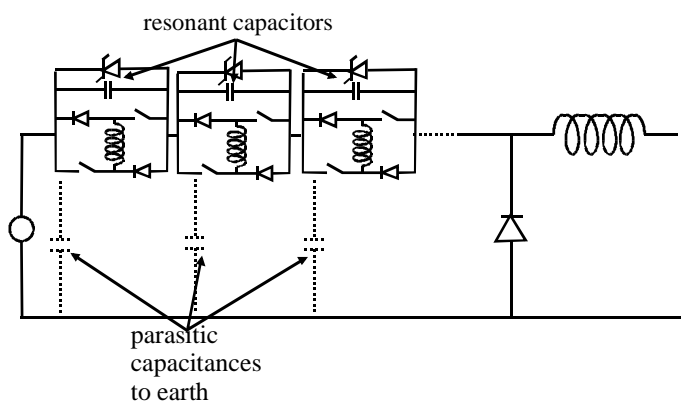

Fig. 10 : Taking into account of energy stored in parasitic capacitances to earth.

\section{REALIZATION}

A converter using 24 stages connected in series was achieved. The switch is designed to commute up to $15 \mathrm{kV}$. The switching frequency is set to $20 \mathrm{kHz}$. The converter is set in an oil tank. This ensures efficient cooling as well as improved dielectric insulation compared to air.

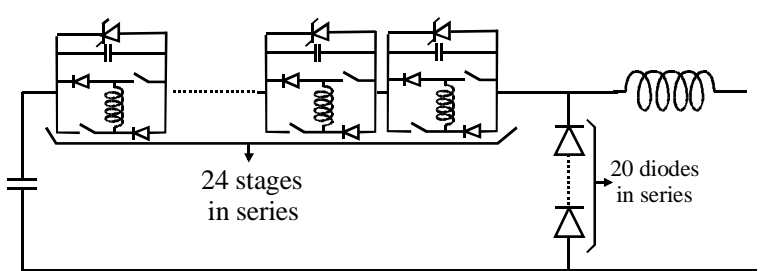

Fig. 11 : Converter realised.

Fig. 12 exhibits a picture of the converter.

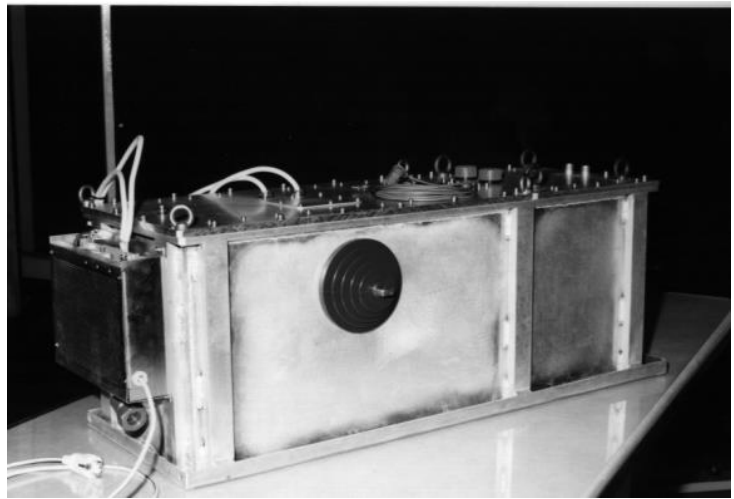

Fig. 12 : Picture of the converter.

\section{High voltage switch}

The high voltage switch is made of 24 stages connected in series. 1200V IGBTs (TO247 case) are used. A $1 \mathrm{kV}$ clamp is provided on every stage by a zener. This protects the stages during off states, like before starting the converter.

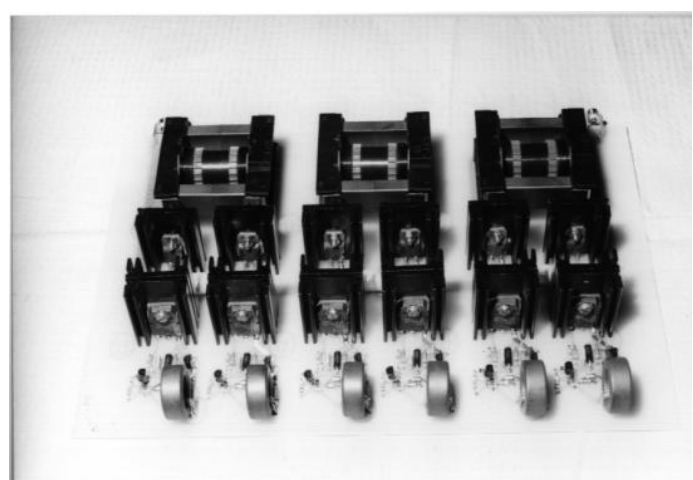

Fig 13 : Picture of a three stages card.

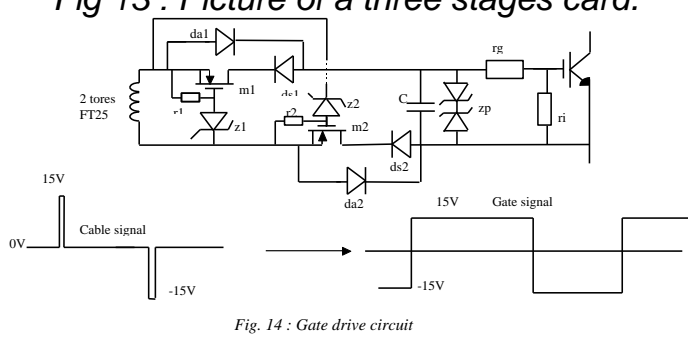

Drive

The high voltage switch needs 48 isolated drives with synchronous trigger pulses. A pulse transformer with a single cable is used. Positive and negative pulses defines on and off gate drive signals. 
Pulses in the cable are bounded to some tens on nanoseconds, owing to the core saturation. To get drive signal of several $\mu$ s long, the system presented in fig. 14 is used. Implementation of the pulse transformer is simple : the cable just cross through the cores (which are in foreground in fig. 13).

A $900 \mathrm{~V}$ inverter (fig. 15) provides on and off pulses.

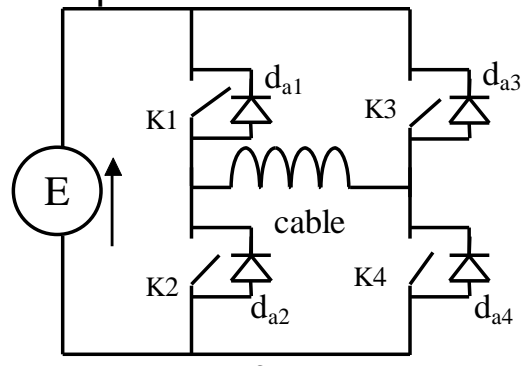

Fig 15 : pulse transformer's inverter.

\section{Freewheel diode}

The freewheel diode is made of 20 serial connected $1200 \mathrm{~V}$ rated diodes. Those diodes are designed to withstand avalanche, making auxiliary sharing voltage system useless.

\section{EXPERIMENTATION}

The converter has been tested as a pulsed load to control the charge of capacitors of a laser power supply (cf. fig.16).

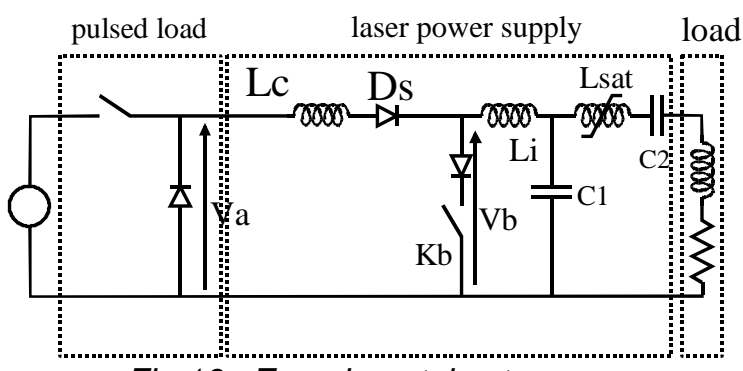

Fig 16 : Experimental set up.

When the pulsed load is switched on, a voltage is applied at the laser power supply input, and a resonant load of $\mathrm{C} 1$ and $\mathrm{C} 2$ occurs (through inductor LC and diode Ds). When pulsed load is switched off, the load of capacitors goes on, but with lower amplitude (then current flows through freewheel diode), until Ds switch off at inductor current's zero. This allows to control with the duty cycle the energy delivered to the laser power supply. Then Kb turns on and invert the voltage across $\mathrm{C} 1$ thanks to a resonance with inductor $\mathrm{Li}$. The consequence is that voltage across Lsat increases until it saturates, allowing the energy stored into $\mathrm{C} 1$ and $\mathrm{C} 2$ to flow through the load.

Fig 17 shows a waveform corresponding to a $9 \mathrm{kV}$ input voltage and a $20 \mathrm{kHz}$ switching frequency. Voltage $\mathrm{Va}$ across the freewheel diode. $9 \mathrm{kV}$ pulses are delivered to the laser power supply. $\mathrm{Vb}$ shows the charge of capacitors, and the switching of $\mathrm{Kb}$.

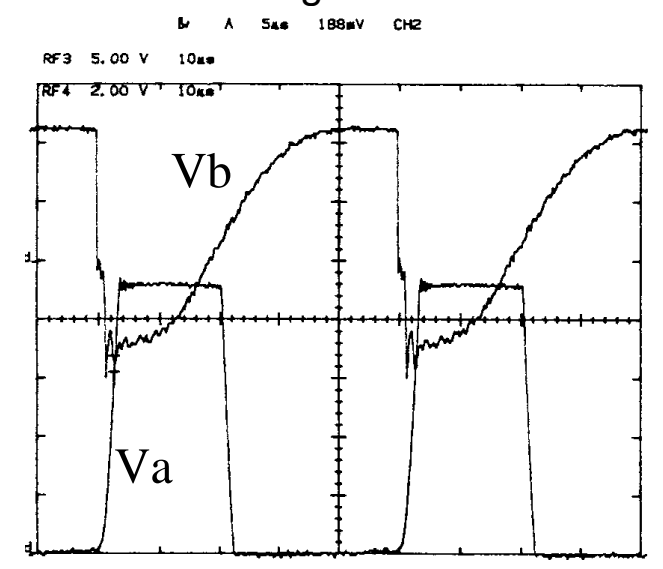

Fig 17: Voltages $\mathrm{Va}(2 \mathrm{kV} / \mathrm{div})$ and $\mathrm{Vb}(5 \mathrm{kV} / \mathrm{div})$.

\section{CONCLUSION}

Soft switching makes the achievement of high voltage chopper easier. The chosen soft switching topology is simple to implement (constant frequency, drive close to classical PWM hard switching) and it is suitable for serial connection, by taking advantage of 
resonant inductors and capacitors distributed among IGBTs. A switch was tested at a $9 \mathrm{kV}$ input voltage.

[1] Y. Chéron, H. Foch, J. Salesses, "Study of a resonant converter using power transistors in a $25 \mathrm{~kW}$ X-rays tube power supply », IEEE PESC Proceedings, 1985.

[2] R. Guidini, "Interrupteurs rapides haute tension réalisés par mise en série de composants semiconducteurs pour convertisseurs de forte énergie", thèse de docteur ingenieur, Montpellier 1995.

[3] Patrick R. Palmer, Anthony N. Githiari, "The series connection of IGBT with optimised voltage sharing in the switching transient", IEEE1995.

[4] R. Letor, "Series connection of MOSFET, bipolar an IGBT devices", SGS-Thomson AN480.

[5] Avelar, Henrique José \& Cortizo, Porfirio Cabaleiro, "Link DC resonant converter without overvoltage: study of a buck converter", EPE Proceedings, 1991.

[6] N. Lapassat, D. Chatroux, D. Lafore, "Serial connection: soft switching, away to reduce parasitic overcurrents ", EPE Proceedings, 1997. 